\title{
Sleep EEG Signatures in COVID-19 Survivors
}

\author{
Abhishek Goyal ${ }^{1}\left[\right.$ Khushboo Saxena $^{1} \cdot$ Avishek Kar $^{1} \cdot$ Parneet Kaur Bhagtana $^{1}$. Chinta Siva Koti Rupa Sridevi ${ }^{1}$. \\ Swanzil Chaudhary ${ }^{1} \cdot$ Rashida Ali $^{1}$
}

Received: 23 August 2021 / Revised: 26 September 2021 / Accepted: 30 September 2021 / Published online: 12 October 2021

(C) The Author(s), under exclusive licence to Springer Nature Singapore Pte Ltd. 2021

\begin{abstract}
Study Objectives Effect of COVID-19 on sleep architecture is not known. This study was done to find out EEG changes seen during sleep in COVID-19 survivors.

Methodology In this prospective single centre study, consecutive patients diagnosed with RTPCR confirmed COVID 19 were included after 4-6 weeks of discharge from hospital. All patients underwent level I PSG. EEG was analysed for presence of abnormal EEG pattern.

Results Total 189 patients were contacted telephonically for participation in this study. Finally 81 patients (55 males, 26 females) underwent Level I PSG. Total sleep time was 345.1 $\pm 85.1 \mathrm{~min}$. Sleep efficiency was 76.0 $\pm 14.2 \%$. Mean time (\%) during N1, N2, N3 and Rapid Eye movement (REM) was 16.4\%, 59.2\%, 7.9\% and 18.4\% percentage, respectively. Mean AHI was $28.7 \pm 22.8$ per hour and arousal index was $23.9 \pm 13.3$. Alpha intrusion was the most common EEG finding $(78 \%)$, followed by cyclical alternating pattern (59\%). REM density was significantly increased in $38 \%$ of patients. REM alpha bursts and increased spindles were also seen in $27 \%$ and $16 \%$.

Conclusion Abnormal EEG waves are very commonly seen in COVID-19 survivors. Presence of these abnormal PSG-EEG waves hints that COVID-19 might have similar effects as depression, insomnia on these subjects, at least in short run. Whether these changes are temporary or permanent needs to be evaluated by performing serial polysomnographies in patients with COVID-19 ARDS.
\end{abstract}

Keywords COVID-19 $\cdot$ Polysomnography $\cdot$ Electroencephalography $\cdot$ REM density $\cdot$ Alpha intrusion $\cdot$ Cyclical alternating pattern

\section{Introduction}

COVID-19 has been associated with residual respiratory and cardiovascular complications even in asymptomatic patients. Case series in patients with COVID-19 have shown that patients can have persistent dyspnoea, restlessness, anxiety, palpitations, fatigability $[1,2]$. Similarly neurological complications like headache, anxiety and insomnia are also reported after discharge [3]. These symptoms were also seen in patients who were asymptomatic at the time of COVID19 infection.

COVID-19 infection is associated with neuropsychiatric complications during infective phase, however, long term consequences are also likely to occur $[4,5]$. In one of the

Abhishek Goyal

abhishek.pulmed@aiimsbhopal.edu.in

1 AIIMS Bhopal, Saket Nagar, Bhopal 462024, India studies done in patients with acute neurological and psychiatric complications of COVID-19 infection; $62 \%$ patients had cerebrovascular accidents and $31 \%$ patients had altered mental status (e.g., psychosis, dementia like syndrome and mood disorders) [4]. A systematic review reported that depression (33\%), anxiety (36\%) and insomnia (41\%) were the common neuropsychiatric findings during acute infection of Severe Acute Respiratory (SARS) and Middle East Respiratory Syndrome (MERS) viral infections, which also belong to coronavirus family [6]. After resolution of acute illness, depressed mood was reported by $11 \%$ patients, anxiety by $12 \%$ patients and similar proportion reported insomnia [6]. However, such data is not available for COVID-19 infected patients. Insomnia, depression and sleep disturbances has been shown to be highly prevalent in patients with COVID-19 but effect of COVID-19 on sleep architecture is not known [7]. 
In this paper, we are reporting EEG changes seen in COVID-19 survivors who underwent Level I Polysomnography (PSG).

\section{Methodology (Fig. 1)}

This study is a sub-analysis of a prospective observational study in which level I Polysomnography was done in COVID 19 survivors [8].

In this prospective single centre study, consecutive patients diagnosed with RTPCR confirmed COVID 19 were included after discharge from hospital. The study was conducted during October-December 2020. Demographic data, past medical history and severity of COVID-19 infection was obtained from hospital records.

Inclusion Criteria Patient meeting all the criteria were included:

1. Age $\geq 18$ years.

2. Patients positive for COVID 19 by RT PCR at the time of infection

3. Patients must have been tested negative for COVID-19 (by nasopharyngeal swab-RT PCR) at least 3 weeks prior to study.

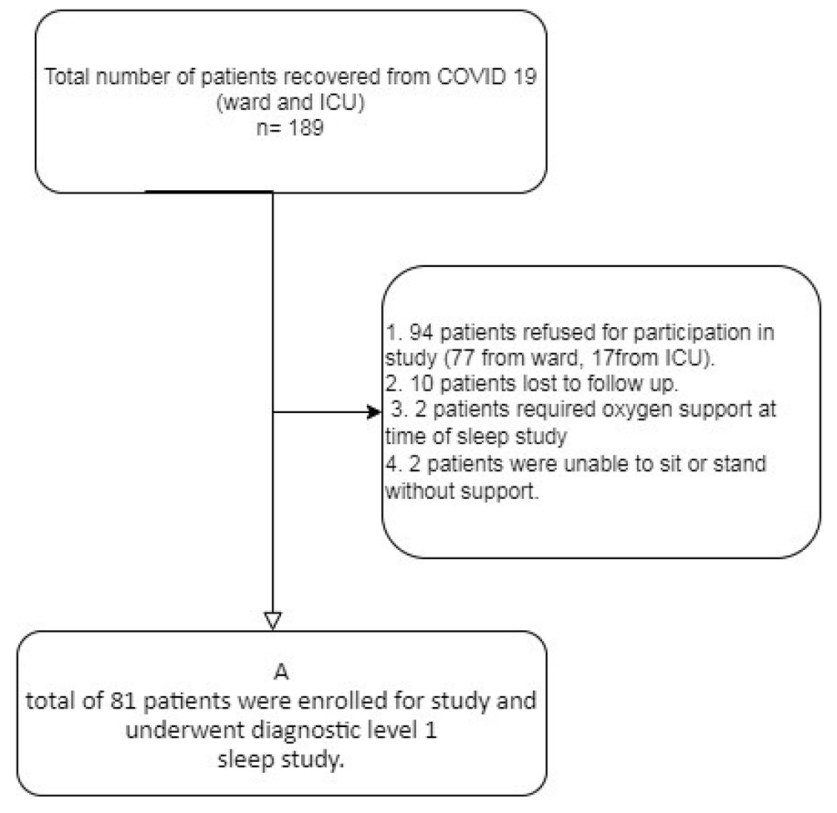

Fig. 1 Flowchart of patients with COVID-19 who underwent level I Polysomnography

\section{Exclusion criteria:}

1. Subjects already taking neuropsychotropic medications e.g., antipsychotics, hypnotics, antidepressants, dopamine agonists (as this can hamper sleep architecture).

2. Subjects with history of neurodegenerative disorders, stroke or any other central nervous system lesion.

3. Patients who refused to give consent for PSG.

4. Patients who were not able to sit and stand without support even after 3 weeks of discharge or were still on oxygen (as it would have been difficult to perform PSG in them).

5. Any symptoms of active pulmonary infection.

During this period, patients with mild severity $\left(\mathrm{spo}_{2}>94 \%\right)$ and moderate-severe disease (who required oxygen supplementation) were being admitted in our hospital. Hospital records were retrieved for consecutive patients who fulfilled both inclusion and exclusion criteria. Appointment for polysomnography was allotted after 3 weeks of negative nasopharyngeal and oropharyngeal COVID-19 RT PCR report.

On the day of PSG, patients were again screened for symptoms suggestive of respiratory infection, fever, dyspnoea, throat pain and oxygen requirement prior to PSG hook-up. All demographic parameters, co morbidities and anthropometric parameters were recorded prior to study. Weight of patient was noted from hospital record on day of admission or OPD slip. Weight was again noted on day of PSG. Weight loss or gain from time of ICU admission was noted for each patient.

\subsection{Precautions taken during Polysomnography}

These measures were taken during PSG in all patients to prevent spread of infection among patients and staff:

1. No attendant was allowed in sleep lab room along with patient.

2. On day of sleep study, temperature and active respiratory symptoms were screened and those with active infection were excluded.

3. Technicians wore PPE (Personal Protective Equipment) as per hospital protocol.

4. Disposable bed covers were used and sleep room was fumigated and locked for $72 \mathrm{~h}$ after PSG.

\subsection{Polysomnography (PSG)}

All patients underwent level I PSG (Philips Respironics Alice 6, USA). Following parameters were measured during PSG: Electroencephalogram (EEG), electro-oculogram, submentalis Electromyogram (EMG), nasal and oral airflow, anterior tibialis EMG, body position and electrocardiogram, 
thoracic and abdominal movements. Scoring of events was done according to AASM scoring manual version 2012 [9]. PSG analysis was performed by sleep medicine residents first and confirmed by sleep physician. EEG was analysed for presence of abnormal EEG pattern. Alpha intrusion, REM alpha bursts, Increased REM density, Cyclical alternating pattern and increased spindles were seen in majority of sample population and is described as following:

\subsection{Alpha intrusion}

When during sleep, there is increased presence of alpha waves, it is known as alpha intrusion. It has been reported in fibromyalgia, depression, chronic fatigue syndrome, anxiety disorder, insomnia, obstructive sleep apnoea, circadian disorders and narcolepsy [10]. Another variant of alpha intrusion is REM alpha bursts (Fig. 2).

\subsection{REM alpha bursts (Fig. 2)}

Human rapid eye movement (REM) sleep is defined by the appearance of mixed-frequency electroencephalographic (EEG) activity concomitantly with rapid eye movements (REMs) and muscular atonia. Studies have shown that alpha bursts are detected with a maximum amplitude over occipital brain regions. This phasic brain activity usually has a shorter duration (at least $3 \mathrm{~s}$ ) than an EEG arousal and is not accompanied by concurrent increase in the submental EMG amplitude and may represent microarousals rather than arousals.
Alpha bursts has been reported in psychiatric disorders like fibromyalgia, depression, chronic fatigue syndrome, anxiety disorder, and primary sleep disorders like psychophysiological insomnia, obstructive sleep apnoea, circadian disorders and narcolepsy [10].

\subsection{REM density (Fig. 3)}

Is usually defined by number of rapid eye movements per epoch of REM sleep. Increased REM density has been reported in patients with depression. Increased REM density has also been associated with poor prognosis in various psychiatric disorders like suicidality in schizophrenia, relapse in recovering alcoholics, and poor response to antidepressant in depression [11].

In this study REM density is defined as the increase in number of eye movements per minute during REM sleep. REM cycles of $<2$ min duration were not included in the analysis. For the purpose of analysis, REM cycles interrupted by $<5$ min of wakefulness or NREM sleep have been considered to be part of the same REM cycle.

\subsection{Sleep spindles}

Sleep spindles are bursts of $11-15 \mathrm{~Hz}$ (sigma frequency band) activity, typically between 0.5 and $2 \mathrm{~s}$ in duration. Spindles are most prominent during N2 sleep and are in fact a defining feature of this stage. They are generated in the thalamic reticular nucleus and synchronized by thalamocortical

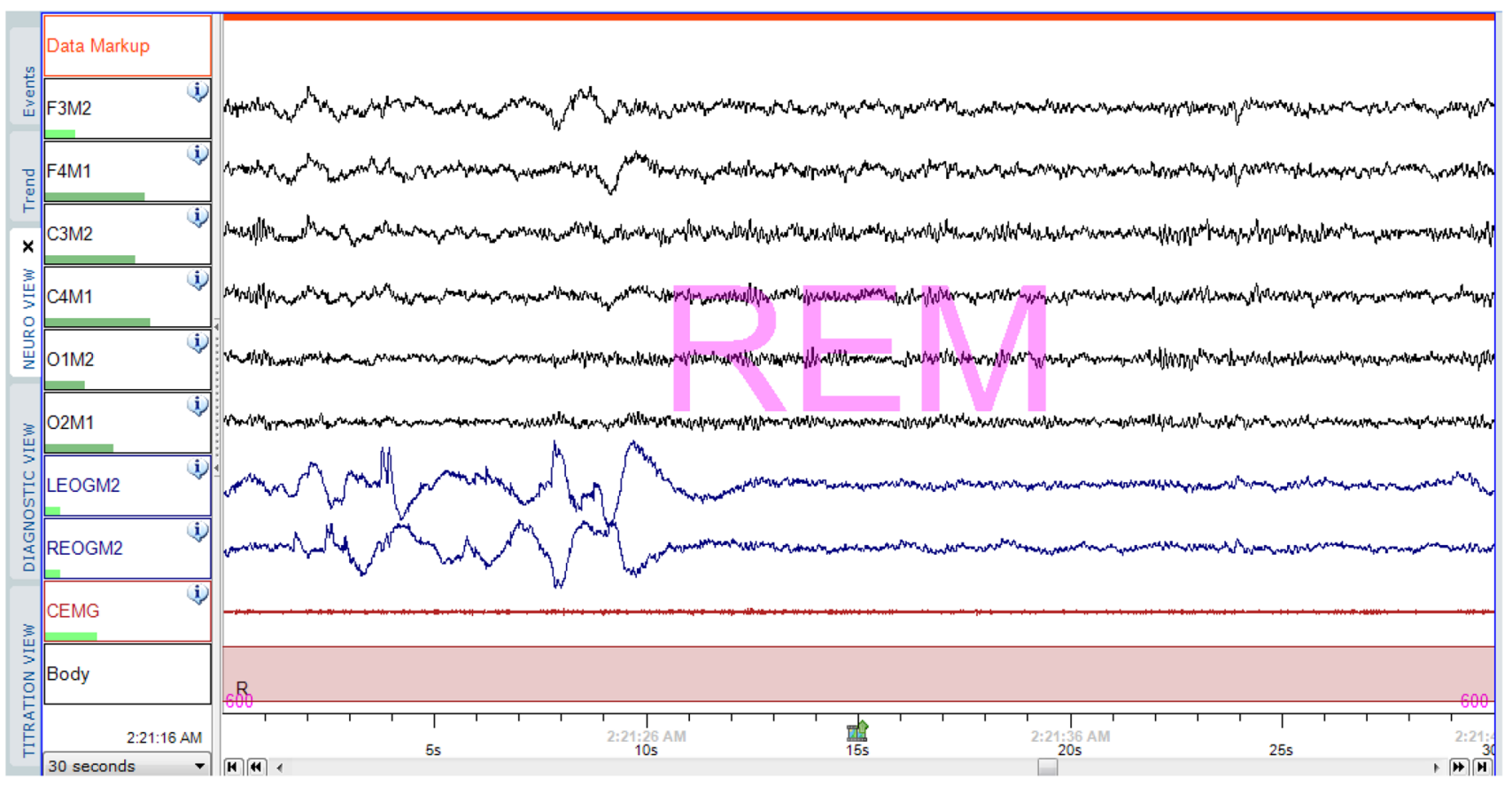

Fig. 2 REM alpha bursts (alpha intrusion in REM without associated increased Chin EMG activity) indicating microarousals 


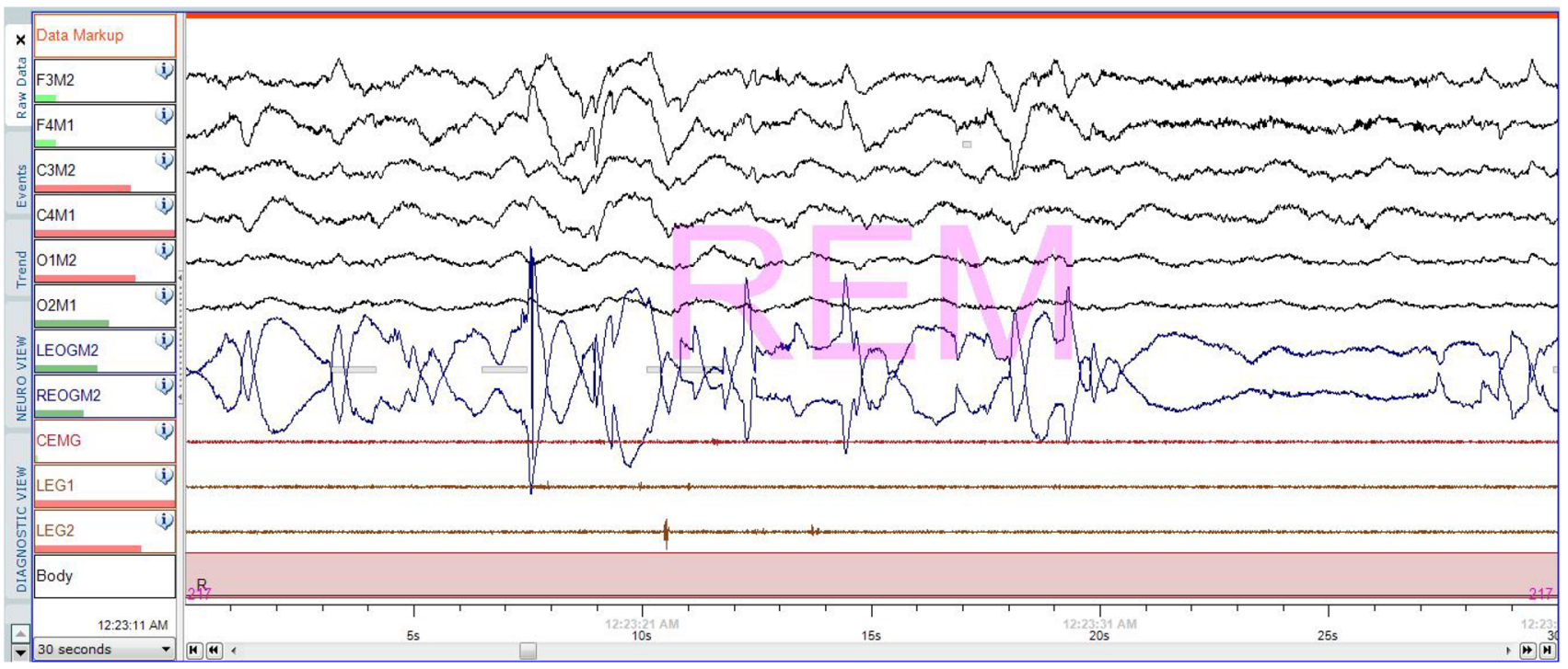

Fig. 3 Figure showing excessive REM movements during REM stage indicating high REM density

interactions. They have important role in normal memory and learning, and are believed to be influenced by dynamic alterations in synaptic plasticity seen during sleep.

Studies have shown excessive spindle density/excessive spindles during $\mathrm{N} 2$ was associated with a greater duration of N2 sleep and shorter duration of N3 sleep (slow wave sleep), indicating it might be associated with sleep instability [12].

\subsection{Cyclic alternating pattern (Fig. 4)}

Cyclic alternating pattern (CAP) is a well-defined marker of the physiological cerebral activity which occurs in conditions involving reduced vigilance like sleep and coma. It is thought to translate a state of arousal instability involving muscle, behavioural, and autonomic functions. During NREM sleep, cyclic alternating pattern is organized in sequences. A cyclic alternating pattern (CAP) sequence is composed of succession of cyclic alternating pattern cycles. A CAP cycle is composed of phase A (lumps of sleep phasic events) followed by phase B (return to EEG background). All CAP sequences begin with a phase A and end with a phase B. Each phase of cyclic alternating pattern is 2-60 s in duration. Cyclic alternating pattern sequences commonly precede the transition from nonREM to REM sleep and end just before REM sleep onset [13]. CAP is considered to be the main expression of sleep

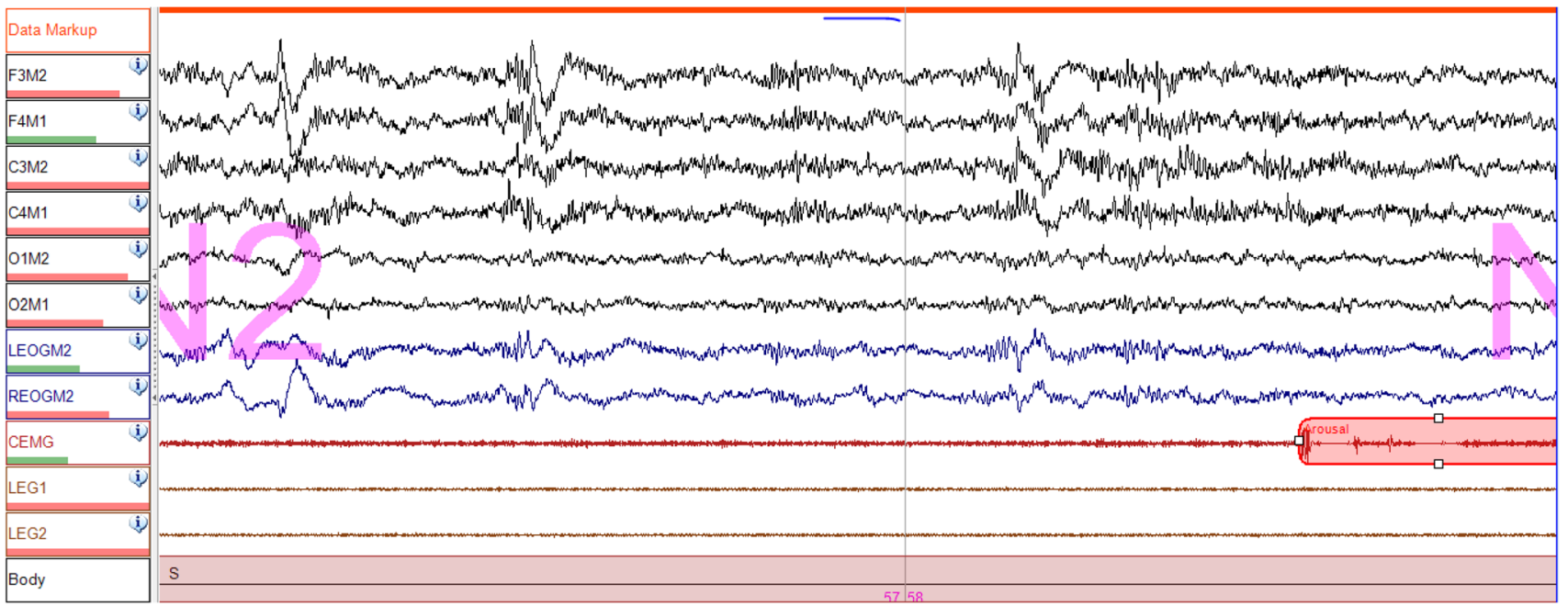

Fig. 4 Cyclical Alternating pattern 
microstructure. High amounts of CAP rate indicate that one or more factors interfere with sleep consolidation. Several studies have shown that sleep disorders such as OSA, PLM, and insomnia have increased CAP measures [14-16].

It is important to note that above mentioned EEG changes have a characteristic pattern and analysis was done subjectively (rather than objectively by methods like spectral density analysis by Fast Fourier Technology). This is one of the important limitations of this study. Final decision on EEG characteristics (like Alpha intrusion, REM alpha bursts, Increased REM density, Cyclical alternating pattern and increased spindles) was done after patterns were verified by three independent and blinded specialist sleep physicians.

\subsection{Ethics review}

Approval for ethics approval was taken approval from AIIMS Bhopal Institutional Human Ethics committee (IHEC-LOP/2020/IM309). Patients were counselled regarding purpose of the study and consent was taken from every patient.

\subsection{Data analysis}

Data analysis was done using R software [17] and gt summary [18] packages. We have summarized nominal variables with count and percentage and numerical variables with mean and its standard deviation. We have stratified our data across those who had severe OSA (defined by AHI $\geq 30$ ) and those with $\mathrm{AHI}<30$. We also stratified patients who required respiratory support (oxygen, NIV/HFNC/IMV) and those who did not require respiratory support. Then we have tested difference in distribution of numerical and categorical variables across these groups by using $t$-test and Chi-square test respectively. $p$-value less than 0.05 was considered as statistically significant.

\section{Results}

Total 189 patients were contacted telephonically for participation in this study. Ninety four patients refused for study, 10 patients lost to follow-up and 4 patients had persistent significant disability. Finally 81 patients (55 male, 26 female) underwent Level I PSG (Fig. 1).

Baseline characteristics (Table 1): Mean Age was $52.6 \pm 10.9$ years and mean BMI at time of PSG was $27.5 \pm 6.2 \mathrm{~kg} / \mathrm{m}^{2}$. (Table 1) Seventeen out of sixty seven had $\mathrm{BMI} \geq 30 \mathrm{~kg} / \mathrm{m}^{2}$ and 50 had BMI $<30 \mathrm{~kg} / \mathrm{m}^{2}$. Mean weight loss of patients was $3.1 \pm 5.5 \mathrm{~kg}$ from time of ICU admission and PSG (Table 2). Six patients had weight gain and 29 had weight loss; rest 32 patients had constant weight (i.e. variation of less than $1 \mathrm{~kg}$ ) between these two timelines.

Out of 81 patients, 14 did not require any kind of oxygen support during their illness and were never hospitalised (Table 3). Sixty seven patients required respiratory support and were hospitalised during COVID-19 infection. Out of these 67 patients, 22 patients required oxygen varying from 5 to 15 LPM and did not require either Non Invasive Ventilation(NIV) or Invasive Mechanical Ventilation (IMV); 32 patients were managed with NIV during ICU stay and 13 patients required IMV. History of Diabetes Mellitus (DM) and hypertension was present in 40 patients each.

Table 1 Baseline demographic and clinical characteristics of study population

\begin{tabular}{|c|c|c|c|c|c|c|}
\hline Characteristic & Overall, $N=81^{\mathrm{a}}$ & $\mathrm{IMV}, n=13^{\mathrm{a}}$ & $\mathrm{NIV}, n=32^{\mathrm{a}}$ & No Oxygen, $n=14^{\mathrm{a}}$ & Oxygen, $n=22^{\mathrm{a}}$ & $p$-value ${ }^{\mathrm{b}}$ \\
\hline Age & $53.3(11.1)$ & $54.4(9.1)$ & $50.6(11.3)$ & $56.8(12.2)$ & $54.4(11.0)$ & 0.215 \\
\hline Men & $55(68 \%)$ & $9(69 \%)$ & $20(62 \%)$ & $9(64 \%)$ & $17(77 \%)$ & 0.724 \\
\hline $\mathrm{DM}$ & $43(53 \%)$ & $8(62 \%)$ & $17(53 \%)$ & $3(21 \%)$ & $15(68 \%)$ & 0.046 \\
\hline HTN & $47(58 \%)$ & $9(69 \%)$ & $19(59 \%)$ & $7(50 \%)$ & $12(55 \%)$ & 0.759 \\
\hline CAD & $7(8.6 \%)$ & $2(15 \%)$ & $4(12 \%)$ & $1(7.1 \%)$ & $0(0 \%)$ & 0.220 \\
\hline BMI & $27.3(5.8)$ & $24.5(3.8)$ & $28.3(7.1)$ & $26.6(3.5)$ & $28.1(5.7)$ & 0.286 \\
\hline Waist circumference & $101.2(14.6)$ & $92.8(22.2)$ & $105.0(14.9)$ & $98.2(6.7)$ & $102.5(10.0)$ & 0.250 \\
\hline Hip circumference & $104.6(12.6)$ & $101.3(8.0)$ & $107.0(16.4)$ & $102.6(7.5)$ & $104.2(10.9)$ & 0.838 \\
\hline Neck circumference & $38.5(5.6)$ & $36.5(11.3)$ & $38.7(4.2)$ & $37.8(3.0)$ & $39.7(3.0)$ & 0.420 \\
\hline NOSAS & $9.5(5.2)$ & $11.8(8.9)$ & $8.6(3.8)$ & $8.0(4.3)$ & $10.4(4.0)$ & 0.223 \\
\hline STOPBANG & $3.7(1.5)$ & $4.3(1.9)$ & $3.8(1.4)$ & $3.0(1.2)$ & $3.6(1.3)$ & 0.224 \\
\hline ESS & $8.3(3.4)$ & $8.8(4.2)$ & $8.1(3.7)$ & 7.4 (3.7) & $9.0(2.2)$ & 0.373 \\
\hline
\end{tabular}

${ }^{\mathrm{a}}$ Statistics presented: Mean (SD); $n(\%)$

${ }^{\mathrm{b}}$ Statistical tests performed: Kruskal-Wallis test; Fisher's exact test; chi-square test of independence 
Table 2 Sleep parameters segregated according to severity of OSA
Table 3 Sleep parameters stratified by no oxygen vs any oxygen

\begin{tabular}{llllr}
\hline Characteristic & Overall, $N=81^{\mathrm{a}}$ & AHI $<30, n=54^{\mathrm{a}}$ & AHI $\geq 30, n=27^{\mathrm{a}}$ & $p$-value \\
\hline Total sleep time & $345.1(85.1)$ & $350.4(76.6)$ & $334.7(100.9)$ & 0.810 \\
Sleep efficiency & $76.0(14.2)$ & $75.6(13.1)$ & $76.8(16.5)$ & 0.286 \\
Sleep latency & $20.7(22.4)$ & $24.6(25.3)$ & $12.9(12.3)$ & 0.025 \\
N1 & $16.4(10.5)$ & $14.6(8.7)$ & $19.9(12.8)$ & 0.071 \\
N2 & $59.2(11.8)$ & $57.9(11.4)$ & $61.8(12.4)$ & 0.277 \\
N3 & $7.9(7.7)$ & $8.6(8.3)$ & $6.5(6.2)$ & 0.428 \\
REM & $18.4(13.9)$ & $21.1(15.0)$ & $13.0(9.5)$ & $<0.001$ \\
Total AHI & $28.7(22.8)$ & $16.0(7.1)$ & $54.3(22.0)$ & $<0.001$ \\
NREM AHI & $27.8(23.8)$ & $14.6(7.7)$ & $54.2(23.3)$ & $<0.001$ \\
REM AHI & $30.0(24.7)$ & $20.3(15.7)$ & $49.3(28.3)$ & $<0.001$ \\
POSA & $40(49 \%)$ & $32(59 \%)$ & $8(30 \%)$ & 0.023 \\
Arousal index & $23.5(14.1)$ & $18.9(8.2)$ & $32.5(18.6)$ & $<0.001$ \\
ODI & $21.9(26.5)$ & $9.7(7.6)$ & $46.3(33.4)$ & $<0.001$ \\
T90 & $5.0(15.9)$ & $1.3(4.7)$ & $12.4(25.5)$ & $<0.001$ \\
Weight loss & $2.5(5.1)$ & $2.3(5.3)$ & $2.9(4.9)$ & 0.573 \\
Alpha intrusion & $60(78 \%)$ & $38(75 \%)$ & $22(85 \%)$ & 0.471 \\
Increased REM density & $30(38 \%)$ & $21(40 \%)$ & $9(33 \%)$ & 0.713 \\
REM alpha burst & $21(27 \%)$ & $13(26 \%)$ & $8(30 \%)$ & 0.942 \\
Cyclical alternating pattern & $46(59 \%)$ & $32(63 \%)$ & $14(52 \%)$ & 0.491 \\
Excessive spindles & $12(16 \%)$ & $10(20 \%)$ & $2(7.7 \%)$ & 0.202 \\
\hline
\end{tabular}

${ }^{a}$ Statistics presented: Mean (SD); $n(\%)$

${ }^{\mathrm{b}}$ Statistical tests performed: Wilcoxon rank-sum test; Fisher's exact test; chi-square test of independence

\begin{tabular}{llllr}
\hline Characteristic & Overall, $N=81^{\mathrm{a}}$ & Room air $(n=14)^{\mathrm{a}}$ & $\begin{array}{l}\text { Oxygen Supplementa- } \\
\text { tion given }(n=67)^{\mathrm{a}}\end{array}$ & $p^{\text {-value }}{ }^{\mathrm{b}}$ \\
\hline Total sleep time & $345.1(85.1)$ & $354.4(83.3)$ & $343.2(86.0)$ & 0.750 \\
Sleep efficiency & $76.0(14.2)$ & $76.3(15.1)$ & $75.9(14.2)$ & 0.886 \\
Sleep latency & $20.7(22.4)$ & $24.8(24.6)$ & $19.8(22.0)$ & 0.368 \\
N1 & $16.4(10.5)$ & $18.4(12.6)$ & $15.9(10.1)$ & 0.389 \\
N2 & $59.2(11.8)$ & $61.6(8.3)$ & $58.7(12.4)$ & 0.435 \\
N3 & $7.9(7.7)$ & $4.7(7.7)$ & $8.5(7.6)$ & 0.014 \\
REM & $18.4(13.9)$ & $15.3(7.9)$ & $19.0(14.8)$ & 0.372 \\
Total AHI & $28.7(22.8)$ & $27.1(27.9)$ & $29.1(21.9)$ & 0.300 \\
Arousal index & $23.5(14.1)$ & $21.2(18.0)$ & $23.9(13.3)$ & 0.183 \\
ODI & $21.9(26.5)$ & $17.4(25.0)$ & $22.8(26.9)$ & 0.200 \\
T90 & $5.0(15.9)$ & $0.4(0.8)$ & $6.0(17.4)$ & 0.039 \\
Weight loss & $2.5(5.1)$ & $0.1(2.5)$ & $3.1(5.5)$ & 0.081 \\
Alpha intrusion & $60(78 \%)$ & $11(79 \%)$ & $49(78 \%)$ & $>0.999$ \\
Increased REM density & $30(38 \%)$ & $6(43 \%)$ & $24(37 \%)$ & 0.911 \\
REM alpha burst & $21(27 \%)$ & $6(46 \%)$ & $15(23 \%)$ & 0.168 \\
CAP & $46(59 \%)$ & $8(57 \%)$ & $38(59 \%)$ & $>0.999$ \\
Excessive spindles & $12(16 \%)$ & $4(29 \%)$ & $8(13 \%)$ & 0.217 \\
\hline
\end{tabular}

${ }^{\text {a }}$ Statistics presented: Mean (SD); $n(\%)$

${ }^{\mathrm{b}}$ Statistical tests performed: Wilcoxon rank-sum test; Fisher's exact test; chi-square test of independence 


\subsection{PSG characteristics (Table 2 \& 3)}

In total 81 patients agreed to be a part of study. Results in regards with prevalence of sleep disorder breathing of subjects with ARDS is described separately [8]. In this paper, we are describing sleep architecture and EEG pattern seen in the patients.

Total sleep time was $345.1 \pm 85.1 \mathrm{~min}$. Sleep efficiency was $76.0 \pm 14.2 \%$. Mean time (\%) during N1, N2, N3 and Rapid Eye movement (REM) was $16.4 \%$, $59.2 \%, 7.9 \%$ and $18.4 \%$ percentage respectively. Mean AHI was $28.7 \pm 22.8$ per hour and arousal index was $23.9 \pm 13.3$. Mild, moderate and severe OSA was seen in 22,30 and 27 subjects respectively and only 2 individuals had AHI $<5$.

Alpha intrusion was highly prevalent in our study and was the most common EEG finding and was seen in $78 \%$ of patients followed by cyclical alternating pattern (59\%). REM density was significantly increased in $38 \%$ of patients. The frequency and amplitude of REM during phasic phase of REM was significantly high compared to individuals with OSA done in pre- COVID-19 era in our sleep lab. REM alpha bursts and increased spindles were also seen in $27 \%$ and $16 \%$. REM without atonia was seen in three patients (Aged 52, 67, 60 years); although neither video PSG nor history was supportive of REM Behaviour Disorder. Prevalence of EEG abnormalities did not differ when patients were segregated by OSA severity or level of respiratory support (Tables $2 \& 3$ ).

\section{Discussion}

In this article we present the first description of polysomnography EEG from a cohort of patients with COVID-19 infection. All patients showed a variety of abnormal EEGs findings, such as CAP, alpha intrusion, increased REM density.

A number of mechanisms have been proposed for long term neuropsychiatric sequel among subjects exposed to SARS-CoV-2 infection. SARS-CoV-2 virus has been isolated from the olfactory bulb as well as respiratory centre in central nervous system in addition to hematogenous spread [19]. Systemic inflammation also increases the permeability of blood brain barrier that allows virus its proteins to enter central nervous system. SARS-CoV-2 preferentially affect prefrontal cortex, basal ganglia and hypothalamus in brain, areas that are important for development of neuropsychiatric symptoms and regulation of sleep [19].

Very few studies have been done to find out effect of viral infection on sleep architecture in preCOVID19 era. Intranasal inoculation with influenza virus in mice increased NREM duration and decrease REM duration [20, 21]. Sleep study in patients with HIV virus have shown normal REM density and normal to reduced REM time [22]. In current study, the predominant sleep stage was N1 and N2; REM duration was slightly decreased and it was more decreased in patients with severe OSA. In a study done in ARDS survivors, similarly REM sleep time and N3 was reduced.

Cyclical alternating pattern (CAP) is usually seen in patients with insomnia and is considered marker of unstable sleep [23]. In CAP, characteristic EEG are recurring transient alpha, clusters of vertex waves, K-complexes, or transient high-amplitude delta or mixed-frequency patterns. It is related to sleep instability and preservation, and is observed even in coma. Similarly alpha intrusion and increased spindles during sleep has been associated with unstable sleep, insomnia, OSA and some psychiatric disorders.

Increased REM density has been associated with depression $[11,24]$. It may be a physiological marker for severity or poor prognosis in other psychiatric disorders, including relapse in recovering alcoholics, suicidality in schizophrenia, and poor response to PSD or interpersonal psychotherapy in depression.

Complications of COVID-19 have a close association with sleep disorders $[8,25]$. Presence of these abnormal PSG-EEG waves hints that COVID-19 might have similar effects as depression, insomnia on these subjects, at least in short run. Whether these changes are temporary or permanent needs to be evaluated by performing serial polysomnography in patients with COVID-19 ARDS.

This is first study to show polysomnographic EEG changes seen in COVID-19 survivors and it opens a whole new dimension to effect of COVID-19 on brain. This will add to ever increasing literature and evidence of complications seen in "Long COVID".

An important limitation of this study was that we did not perform spectral density to characterize EEG characteristics in this study.

\section{Conclusion}

Abnormal EEG waves are very commonly seen during sleep in COVID-19 survivors. Presence of these abnormal PSGEEG waves hints that COVID-19 might have effects similar to depression and insomnia, at least in short run. Whether these changes are temporary or permanent needs to be evaluated by performing serial polysomnography in patients with COVID-19 ARDS.

\section{Declarations}

Conflict of interest AG has no conflicts of interest. AK has no conflicts of interest. KS has no conflicts of interest. PKB has no conflicts 
of interest. CSKRS has no conflicts of interest. SC has no conflicts of interest. RA has no conflicts of interest.

\section{References}

1. Carfì A, Bernabei R, Landi F, for the Gemelli Against COVID-19 Post-Acute Care Study Group. Persistent symptoms in patients after acute COVID-19. JAMA. 2020;324(6):603.

2. Logue JK, Franko NM, McCulloch DJ, McDonald D, Magedson A, Wolf CR, et al. Sequelae in adults at 6 months after COVID-19 infection. JAMA Netw Open. 2021;4(2):e210830.

3. Wijeratne T, Crewther S. Post-COVID-19 Neurological Syndrome (PCNS); a novel syndrome with challenges for the global neurology community. J Neurol Sci. 2020;419:117179.

4. Varatharaj A, Thomas N, Ellul MA, Davies NWS, Pollak TA, Tenorio EL, et al. Neurological and neuropsychiatric complications of COVID-19 in 153 patients: a UK-wide surveillance study. The Lancet Psychiatry. 2020;7(10):875-82.

5. Butler M, Pollak TA, Rooney AG, Michael BD, Nicholson TR. Neuropsychiatric complications of covid-19 From acute delirium to long term fatigue, covid-19 has serious neuropsychiatric effects. BMJ. 2020;371:m3871.

6. Rogers JP, Chesney E, Oliver D, Pollak TA, McGuire P, Fusar-Poli $\mathrm{P}$, et al. Psychiatric and neuropsychiatric presentations associated with severe coronavirus infections: a systematic review and metaanalysis with comparison to the COVID-19 pandemic. The Lancet Psychiatry. 2020;7(7):611-27.

7. Xu F, Wang X, Yang Y, Zhang K, Shi Y, Xia L, et al. Depression and insomnia in COVID-19 survivors: a cross-sectional survey from Chinese rehabilitation centers in Anhui province. Sleep Med. 2021. https://doi.org/10.1016/j.sleep.2021.02.002.

8. Goyal A, Saxena K, Kar A, Khurana A, Bhagtana PK, Sridevi CSKR, Pakhare A. Obstructive sleep apnea is highly prevalent in COVID19 related moderate to severe ARDS survivors: findings of level I polysomnography in a tertiary care hospital. Sleep Med. 2021;S1389-9457(21)00350-6.

9. Berry RB, Budhiraja R, Gottlieb DJ, Gozal D, Iber C, Kapur VK, et al. Rules for scoring respiratory events in sleep: update of the 2007 AASM Manual for the Scoring of Sleep and Associated Events. Deliberations of the Sleep Apnea Definitions Task Force of the American Academy of Sleep Medicine. J Clin Sleep Med. 2012;8(5):597-619.

10. Cantero JL, Atienza M. Alpha burst activity during human REM sleep: descriptive study and functional hypotheses. Clin Neurophysiol. 2000;111(5):909-15.

11. Clark C, Dupont R, Golshan S, Gillin JC, Rapaport MH, Kelsoe JR. Preliminary evidence of an association between increased REM density and poor antidepressant response to partial sleep deprivation. J Affect Disord. 2000;59(1):77-83.
12. Purcell SM, Manoach DS, Demanuele C, Cade BE, Mariani S, Cox R, et al. Characterizing sleep spindles in 11,630 individuals from the National Sleep Research Resource. Nat Commun. 2017;8(1):15930.

13. Parrino L, Ferri R, Bruni O, Terzano MG. Cyclic alternating pattern (CAP): the marker of sleep instability. Sleep Med Rev. 2012;16(1):27-45.

14. Korkmaz S, Bilecenoglu NT, Aksu M, Yoldas TK. Cyclic alternating pattern in obstructive sleep apnea patients with versus without excessive sleepiness. sleep disorders. 2018; p. e8713409.

15. Parrino L, Milioli G, De Paolis F, Grassi A, Terzano MG. Paradoxical insomnia: the role of CAP and arousals in sleep misperception. Sleep Med. 2009;10(10):1139-45.

16. Thomas RJ. Cyclic alternating pattern and positive airway pressure titration. Sleep Med. 2002;3(4):315-22.

17. R Core Team. R: A Language and environment for statistical computing [Internet]. Vienna, Austria: R Foundation for Statistical Computing; 2019. https://www.R-project.org/. Accessed 17 Oct 2020.

18. Sjoberg DD, Curry M, Hannum M, Whiting K, Zabor EC, Drill E, et al. gtsummary: Presentation-ready data summary and analytic result tables [Internet]; 2020. https://CRAN.R-project.org/packa ge $=$ gtsummary. Accessed 17 Oct 2020.

19. Banerjee D, Viswanath B. Neuropsychiatric manifestations of COVID-19 and possible pathogenic mechanisms: insights from other coronaviruses. Asian J Psychiatr. 2020;54:102350.

20. Ibarra-Coronado EG, Pantaleón-Martínez AM, Velazquéz-Moctezuma J, Prospéro-García O, Méndez-Díaz M, Pérez-Tapia M, et al. The bidirectional relationship between sleep and immunity against infections. J Immunol Res. 2015;2015:678164.

21. Fang J, Tooley D, Gatewood C, Renegar KB, Majde JA, Krueger JM. Differential effects of total and upper airway influenza viral infection on sleep in mice. Sleep. 1996;19(4):337-42.

22. Wiegand M, Möller AA, Schreiber W, Krieg JC, Fuchs D, Wachter $\mathrm{H}$, et al. Nocturnal sleep EEG in patients with HIV infection. Eur Arch Psychiatry Clin Neurosci. 1991;240(3):153-8.

23. Terzano MG, Parrino L. Origin and significance of the cyclic alternating pattern (CAP). Sleep Med Rev. 2000;4(1):101-23.

24. Goyal A, Kar A, Saxena K. COVID eyes: REM in COVID19 Survivors. Sleep Vigil. 2021. https://doi.org/10.1007/ s41782-020-00124-2.

25. Kar A, Saxena K, Goyal A, Pakhare A, Khurana A, Saigal S, et al. Assessment of obstructive sleep apnea in association with severity of COVID-19: a prospective observational study. Sleep Vigil. 2021;5:1-8.

Publisher's Note Springer Nature remains neutral with regard to jurisdictional claims in published maps and institutional affiliations. 